MITSUBISHI ELECTRIC RESEARCH LABORATORIES

https://www.merl.com

\title{
Blind Multi-path Elimination by Sparse Inversion in Through-the-Wall-Imaging
}

\author{
Mansour, H.; Liu, D. \\ TR2013-120 December 15, 2013
}

\begin{abstract}
In this paper, we propose a multi-path elimination by sparse inversion (MESI) algorithm that removes the clutter induced by internal wall reflections in a Through-the-Wall-Imaging (TWI) system without prior knowledge of the scene geometry. Our approach iteratively recovers the time-domain primary impulse responses of targets behind the front wall then finds a delay convolution operator that best maps the primary impulse response of each target to the multi-path reflections available in the received signal. Since the number of targets and the number of reflecting surfaces is typically much smaller than the downrange extent of the scene, we employ 11 regularized sparse recovery in both the target detection and reflection operator estimation. Moreover, we specify extensions of the MESI algorithm that allow for the detection of targets directly in the image domain even from randomly sub-sampled arrays and compensate for the distortion of the source waveform due to the front wall propagation. We present numerical simulations that demonstrate the effectiveness of MESI in locating targets inside a room with unknown dimensions or wall parameters and highlight the robustness of our scheme to severe measurement noise.
\end{abstract}

IEEE International Workshop on Computational Advances in Multi-Sensor Adaptive Processing

(C) 2013 MERL. This work may not be copied or reproduced in whole or in part for any commercial purpose. Permission to copy in whole or in part without payment of fee is granted for nonprofit educational and research purposes provided that all such whole or partial copies include the following: a notice that such copying is by permission of Mitsubishi Electric Research Laboratories, Inc.; an acknowledgment of the authors and individual contributions to the work; and all applicable portions of the copyright notice. Copying, reproduction, or republishing for any other purpose shall require a license with payment of fee to Mitsubishi Electric Research Laboratories, Inc. All rights reserved. 



\section{Blind Multi-path Elimination by Sparse Inversion in Through-the-Wall-Imaging}

\author{
Hassan Mansour \\ Mitsubishi Electric Research Laboratories \\ Cambridge, MA 02139 \\ Email: mansour@merl.com
}

\author{
Dehong Liu \\ Mitsubishi Electric Research Laboratories \\ Cambridge, MA 02139 \\ Email: liudh@merl.com
}

\begin{abstract}
In this paper, we propose a multi-path elimination by sparse inversion (MESI) algorithm that removes the clutter induced by internal wall reflections in a Through-the-WallImaging (TWI) system without prior knowledge of the scene geometry. Our approach iteratively recovers the time-domain primary impulse responses of targets behind the front wall then finds a delay convolution operator that best maps the primary impulse response of each target to the multi-path reflections available in the received signal. Since the number of targets and the number of reflecting surfaces is typically much smaller than the downrange extent of the scene, we employ $\ell_{1}$ regularized sparse recovery in both the target detection and reflectionoperator estimation. Moreover, we specify extensions of the MESI algorithm that allow for the detection of targets directly in the image domain even from randomly subsampled arrays and compensate for the distortion of the source waveform due to the front wall propagation. We present numerical simulations that demonstrate the effectiveness of MESI in locating targets inside a room with unknown dimensions or wall parameters and highlight the robustness of our scheme to severe measurement noise.
\end{abstract}

Keywords-Through-the-wall-imaging, multi-path elimination, sparse recovery, compressed sensing, blind deconvolution

\section{INTRODUCTION}

Through-the-wall-imaging (TWI) is a technology that allows for the detection of objects inside enclosed structures [1]. In TWI, a source emits an electromagnetic (EM) radar pulse which propagates through the outside wall of the structure, reflects off the internal targets then propagates back to a receiver antenna array [2]. However, depending on the dielectric permittivity and permeability of the walls surrounding the targets, the received signal is often corrupted with indirect secondary reflections from the targets off of the internal walls which result in ghost artifacts in the image domain.

Wall clutter mitigation techniques attempt to eliminate ghost artifacts that arise from the multi-path reflections in TWI. In [3] and [4], multi-path signal models are derived to associate and map the multi-path ghosts to the true target locations, thereby improving the radar system performance by reducing false positives in the original SAR image. A physics based approach to multi-path exploitation is proposed in [5] where the imaging kernel of the back-projection method is designed to focus specific propagation paths of interest. Recently, target sparsity in TWI systems has been utilized for multi-path elimination specifically in compressive sensing synthetic aperture radar (SAR) architectures [6]. The approach incorporates the sources of multi-path reflections of interest into a sparsifying dictionary and solves a group sparse recovery problem to locate the targets from randomly subsampled, frequency stepped SAR data. However, all of the above techniques assume perfect knowledge of the reflective geometry of the scene, which is not necessarily feasible in practice.

In this paper, we propose a new algorithm for multipath removal in physical aperture and SAR TWI that does not require any prior knowledge of the scene geometry. In Section II we model the received signal at the radar array as the sum of a primary response corresponding to the direct path from the target objects, and a multiples' response due to the indirect multi-path reflections. Our approach relies on the assumption that the scene is sparse in the image domain which in turn translates to a sparse time-domain representation of the primary response. Section III presents the proposed multi-path elimination by sparse inversion (MESI) algorithm. MESI first identifies the strongest impulse response of the targets behind the wall and attributes that response to a primary target. Second, a delay operator is computed that matches the primary response to similar reflections in the residual data. Third, the source waveform is updated to compensate for any distortions that may arise from the EM propagation through the wall. The three stages are then repeated until convergence. We also extend the MESI algorithm to perform the recovery in the image domain by introducing an imaging operator in the first primary detection stage. Since the image domain is incoherent with the time domain, the MESI algorithm can therefore recover target objects and eliminate artifacts even from compressive radar arrays where a random subset of the receivers are active. Finally, we demonstrate experimentally in section IV the effectiveness of our MESI algorithm in eliminating the effect of multi-path reflections from an FDTD simulated TWI scene with multiple targets. We also illustrate the robustness of MESI to severe measurement noise as well as its interpolative capability when the receiver array is randomly subsampled.

\section{SIGNAL MODEL}

We consider a monostatic physical aperture radar with a single source located in the center of a one-dimensional array of $n_{r}$ receiving antennas. The radar array is placed parallel to the external front wall. Let $s(t)$ be the time-domain waveform of the pulse that is transmitted by the source, and denote by $g_{p}(t, n)$ the primary impulse response of the scene at each receiver $n \in\left\{1, \ldots n_{r}\right\}$ with walls but excluding multi-path reflections, which is essentially a delayed version of impulse response when there are no walls. Also denote by $g_{m}(t, n)$ 
the impulse response of the multi-path reflections due to the wall clutter as well as other reflecting surfaces in the scene. Using a standard time-domain representation of the received signal model, we express the received signal $r(t, n)$ as follows

$$
r(t, n)=s(t) *\left(g_{p}(t, n)+g_{m}(t, n)\right),
$$

where $*$ is the convolution operator.

Without loss of generality, suppose that there are $K$ target objects in the scene, each inducing a primary impulse response $g_{k}(t, n)$ where the index $k \in\{1 \ldots K\}$. The multiples' impulse response can then be modeled by the convolution of a delay operator $d_{k}(t)$ with the primary impulse response $g_{k}(t, n)$ of each target object in the scene, such that,

$$
g_{p}(t, n)=\sum_{k=1}^{K} g_{k}(t, n), \quad g_{m}(t, n)=\sum_{k=1}^{K} d_{k}(t) * g_{k}(t, n) .
$$

Here we define the delay operator as a sequence of weighted Dirac delta functions

$$
d_{k}(t)=\sum_{i \in \Lambda_{k}} w\left(t_{i}\right) \delta\left(t-t_{i}\right),
$$

where $t_{i}$ is the extra time taken by the multiple to reach the receiver from the $i$ th multi-path source, $w\left(t_{i}\right)$ is the attenuation weight of the $i$ th path, and $\Lambda_{k}$ is the set of all sources of multi-path reflections contributing to the multiple repetitions of object $k$. Consequently, the received signal model can be written as the superposition of the primary and multiple responses of all $K$ objects in the scene as follows

$$
r(t, n)=\sum_{k=1}^{K} s(t) *\left(g_{k}(t, n)+d_{k}(t) * g_{k}(t, n)\right) .
$$

In a blind multi-path elimination scenario, we have no information about the geometry of the scene, the sources and/or paths of the multiple reflections, or the number of sources present in the scene. Our objective is to find the impulse responses $g_{k}(t, n)$ of all $K$ objects using only the received measurements $r(t, n)$ and an estimate of the source waveform $s(t)$. It is obvious from (3) that the inverse problem is non-convex and generally ill-posed. However, we make the following general assumptions that render the problem wellbehaved:

A1. The primary reflections with the most direct path between the receiver and the target objects have the strongest response compared to that of the corresponding multi-path reflections.

A2. The primary reflectors in the scene to be imaged and the number of reflecting surfaces that induce the multi-path are sparse.

\section{MULTIPATH ELIMINATION BY SPARSE INVERSION}

We formulate the multi-path elimination problem as that of finding the primary impulse responses $g_{k}$ and the delay operators $d_{k}$ given the observed raw data $r$ and source waveform $s$. Define the forward model $f($.$) as$

$$
f\left(g_{k}, d_{k}, s\right):=s *\left(g_{k}+d_{k} * g_{k}\right) .
$$

Therefore, we wish to solve the least-squares inverse problem

$$
\min _{g_{k}, d_{k} \forall k \in\{1 \ldots K\}}\left\|r-\sum_{k} f\left(g_{k}, d_{k}, s\right)\right\|_{2} .
$$

However, the function $f($.$) is non-convex in g_{k}$ and $d_{k}$, and the inverse problem is ill-posed in general.

To make the problem well-posed, we introduce sparsity constraints on $g_{k}$ and $d_{k}$ and tackle the non-convexity of $f$ in the variable space by following a block coordinate-descent minimization which renders the problem convex in each of the variables $g_{k}$ and $d_{k}$ separately. We call our approach multipath elimination by sparse inversion (MESI) and present it in generalized form in Algorithm 1.

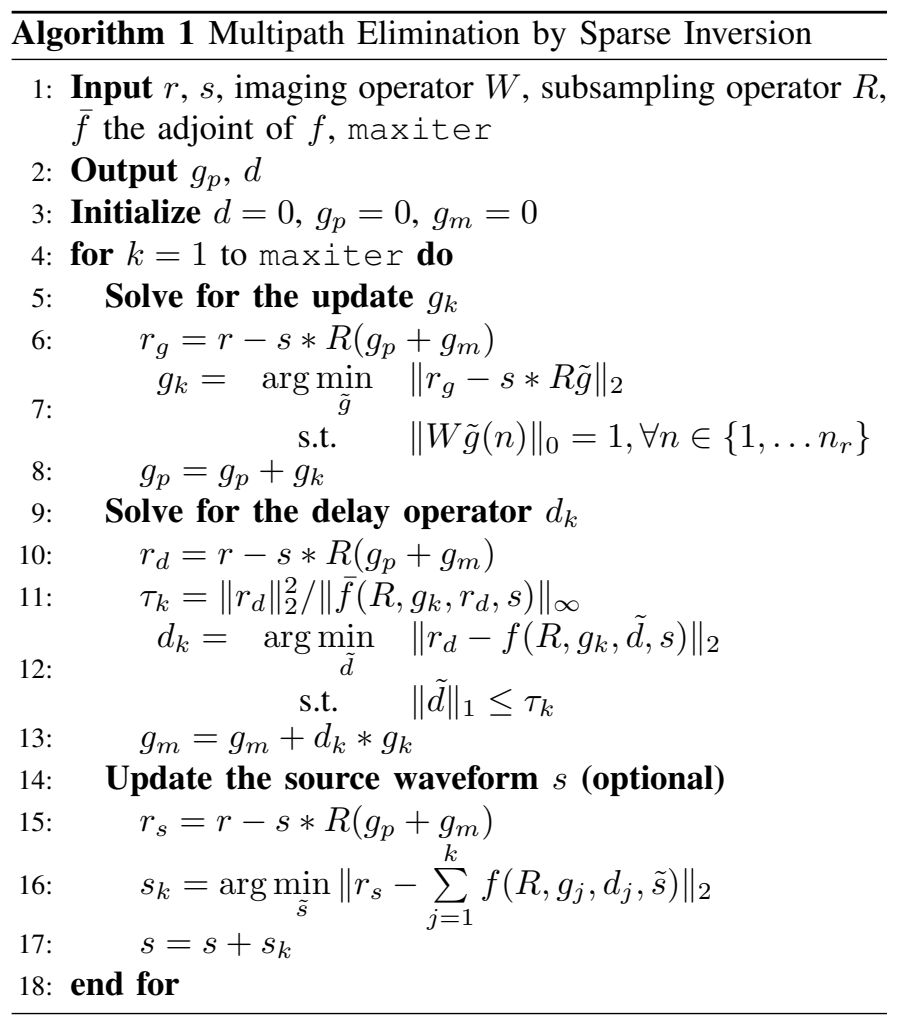

\section{A. The basic MESI algorithm}

The MESI algorithm is separated into two main minimization stages. The first stage constitutes a sparse matched filtering step in which the impulse response $g_{k}$ of the strongest reflector in the residual downrange $r_{g}$ of all receivers is detected. The residual downrange is given by $r_{g}=r-s *\left(g_{p}+g_{m}\right)$, where $g_{p}$ and $g_{m}$ are the estimated primary and multiple impulse responses, both initialized to zero. Following from assumption $\mathrm{A} 1$, we consider $g_{k}$ to be the primary impulse response of a target object in the scene. The primary impulse response update $g_{k}$ identifies a single spike for each receiver that best approximates the data residual $r_{g}$ by solving the sparse recovery problem

$$
\begin{aligned}
g_{k}= & \arg \min _{\tilde{g}}\left\|r_{g}-s * \tilde{g}\right\|_{2} \\
& \text { subject to }\|\tilde{g}(n)\|_{0}=1, \forall n \in\left\{1, \ldots n_{r}\right\} .
\end{aligned}
$$

The primary impulse response is then updated such that $g_{p}=$ $g_{p}+g_{k}$. This first stage of our algorithm parallels the CLEAN algorithm introduced by Högbom in [7] for the identification and deconvolution of point sources, although our algorithm is not based on CLEAN.

In the second stage, we find a delay operator $d_{k}$ that matches the detected impulse response $g_{k}$ with the remaining 
reflections in the data residual $r_{d}=r-s *\left(g_{p}+g_{m}\right)$. Here we assume that all receivers view the multiple reflections of a primary target $g_{k}$ with the same delay operator $d_{k}$. Moreover, assumption A2 indicates that the operator $d_{k}$ should be sparse, which leads to the following LASSO problem

$$
d_{k}=\arg \min _{\tilde{d}}\left\|r_{d}-f\left(g_{k}, \tilde{d}, s\right)\right\|_{2} \text { subject to }\|\tilde{d}\|_{1} \leq \tau_{k}
$$

where $\tau_{k}=\frac{\left\|r_{d}\right\|_{2}^{2}}{\left\|\bar{f}\left(g_{k}, r_{d}, s\right)\right\|_{\infty}}$, and $\bar{f}$ denotes the adjoint of $f$. The choice of $\tau_{k}$ ensures that the delay operator $d_{k}$ contains only a small number of nonzero entries. Therefore, the nonzero components in $d_{k}$ have to match $g_{k}$ with the strongest coherent multi-path reflections in $r_{g}$ while ignoring incoherent responses and noise artifacts. The multiple impulse response $g_{m}$ is then updated according to $g_{m}=g_{m}+d_{k} * g_{k}$. The above two stages are repeated until the maximum number of iterations is reached or a preset data mismatch is reached.

\section{B. Extensions}

The structure of the MESI algorithm allows for direct extensions that address multi-path elimination under a variety of conditions.

For example, one can exploit the sparsity in the image domain instead of the time domain by introducing an imaging operator into the regularization term in (6). Let $W$ be any linear operator that maps the time domain received signal to the down-range/cross-range image pixel domain, i.e.

$$
W: \mathbb{C}^{n_{t} \times n_{r}} \rightarrow \mathbb{C}^{N x \times N y},
$$

where $n_{t}$ is the number of time samples, $N_{x}$ is the resolution in the cross-range, and $N_{y}$ is the resolution in the downrange.

Moreover, since the image domain is incoherent with the time domain, performing the recovery in the image domain allows for the use of MESI in the compressed sensing regime where the receiving antennas are randomly subsampled using a sampling operator $R$ that selects a subset $m$ of the $n_{r}$ receivers, i.e.

$$
R:\left\{1, \ldots n_{r}\right\} \rightarrow \Omega \subseteq\left\{1, \ldots n_{r}\right\}, \quad|\Omega|=m \leq n_{r} .
$$

The generalized sparse recovery problem becomes

$$
\begin{aligned}
& g_{k}=\arg \min _{\tilde{g}}\left\|r_{g}-s * R \tilde{g}\right\|_{2} \\
& \text { subject to }\|W \tilde{g}(n)\|_{0}=1, \quad \forall n \in\left\{1, \ldots n_{r}\right\} \text {, }
\end{aligned}
$$

and the forward model $f($.$) is rewritten as$

$$
f\left(R, g_{k}, d_{k}, s\right):=s * R\left(g_{k}+d_{k} * g_{k}\right) .
$$

Finally, we note that the source waveform $s(t)$ can undergo distortions due to the propagation of the EM wave through dielectric walls. As a result, the received signal at the antenna array is composed of the convolution of the primary and multiple impulse responses with a modified waveform $\tilde{s}(t)$. Depending on the severity of the waveform distortion, the impulse response estimation by matched-filtering stage may exhibit artifacts.

We compensate for waveform distortions by adding a third source waveform estimation stage to the MESI algorithm. In this stage, we compute a least-squares update $s_{k}$ for the source waveform by finding a least-squares fit between the forward model and the data residual as follows

$$
s_{k}=\arg \min _{\tilde{s}}\left\|r_{s}-\sum_{j=1}^{k} f\left(g_{j}, d_{j}, \tilde{s}\right)\right\|_{2} .
$$

We then use the updated source waveform $s=s+s_{k}$ in the subsequent iterations of the MESI algorithm.

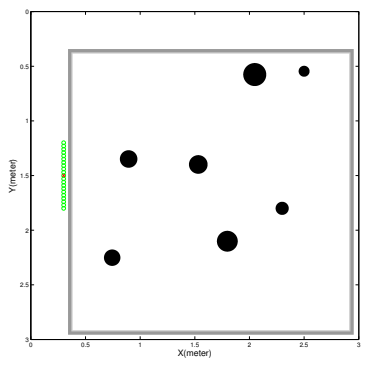

Fig. 1: Schematic of the simulation layout.
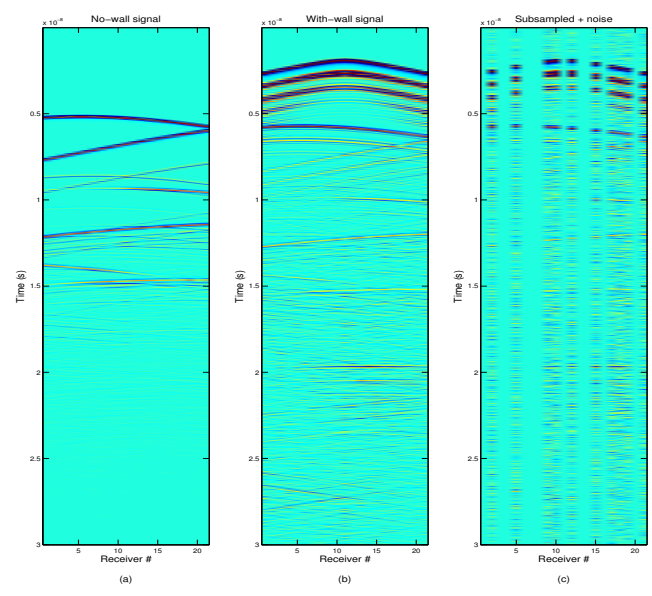

Fig. 2: Received time domain radar reflections of the scene (a) without the wall, (b) with a two layer wall, (c) with the wall, subsampled receiver array, and additive measurement noise resulting in a $5.4 \mathrm{~dB}$ channel.

\section{NUMERICAL EXPERIMENTS}

To study the performance of our algorithm, we generate a $2.7 \mathrm{~m} \times 2.7 \mathrm{~m}$ room with 7 cylindrical targets placed as shown in Fig. 1. A 21-element radar array is placed outside the front (west) wall of the room with a standoff distance equal to $4.5 \mathrm{~cm}$. The wall has two layers. The thickness and relative permittivity are $3 \mathrm{~cm}$ and $\epsilon_{r}=10$ for the outer layer and $1.2 \mathrm{~cm}$ and $\epsilon_{r}=5$ for the inner layer, respectively. The radar transmitter is located at the center of the array and the 21 receiving elements are half-wavelength apart, which is $3 \mathrm{~cm}$ for the pulse central frequency $f_{c}=5 \mathrm{GHz}$. We then use a twodimensional FDTD simulator to emit a derivative of Gaussian pulse from the transmitter and record the EM reflections at all 21 receivers. Fig. 2 illustrates the time-domain signal received at the antenna array when (a) no walls are present, (b) two layer walls are added to the perimeter of the room, and (c) when the walls are present, the channel is noisy at $5.4 \mathrm{~dB}$ and the receiver array is randomly subsampled such that half of the receiving antennas are switched off. 


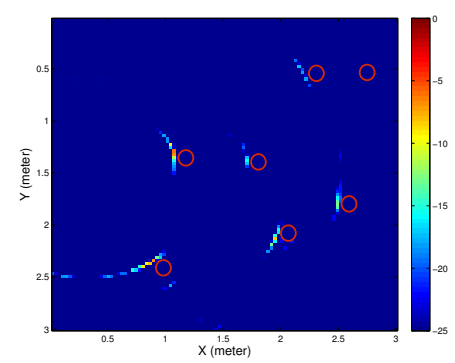

(a)

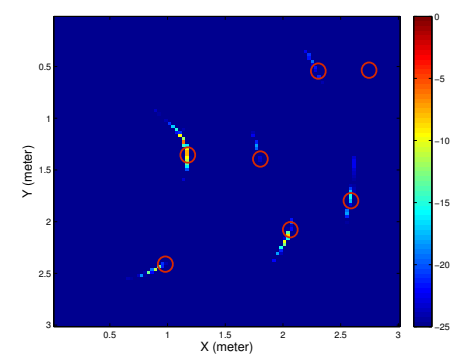

(d)

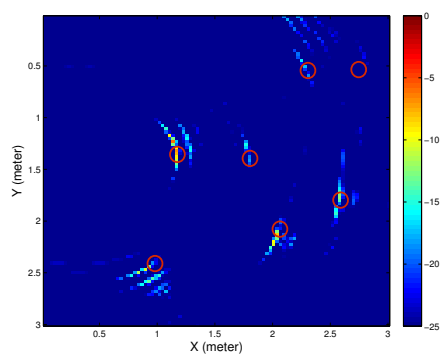

(b)

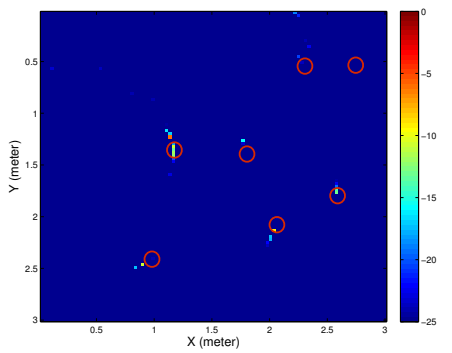

(e)

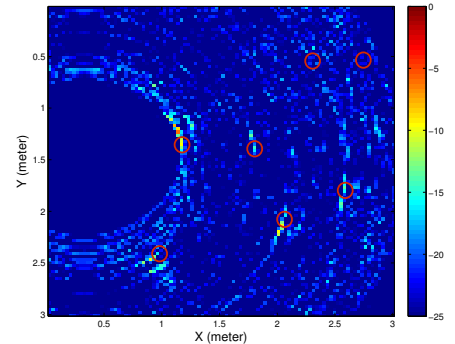

(c)

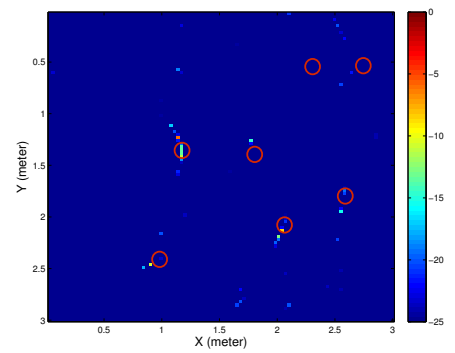

(f)

Fig. 3: Result of applying a beam forming operator to image the time domain data in Fig. 2 from (a) the no-wall signal, (b) the with-wall signal, (c) the with wall signal contaminated with noise and subsampled receivers. The images after multi-path elimination and denoising using MESI are shown in the bottom row for the solution (d) using time-domain sparsity, (e) using image-domain sparsity, and (f) using image domain sparsity to recover from noise and subsampling. The red circles identify the true locations of the targets. All images are shown in logarithmic scale.

Notice that the radar reflections in Fig. 2a still contain some multi-path reflections from the other targets in the scene even though there are no walls present. Moreover, the response of the target in the top right corner of the room is completely missing from the data due to the occlusion by its adjacent target. The multi-paths are clearly visible in Fig. $2 \mathrm{~b}$ as a result of the walls of the room. In performing the recovery, we first time-gate the received signals in order to mask the direct front-wall reflections. Fig. $3 a$ to $3 \mathrm{c}$ show the result from beam forming the time-domain data shown in Fig. 2a to $2 c$, respectively. Notice that the beamformed images exhibit a spatial shift due to the wall propagation and contain ghost targets. Multi-path reflections from the top wall are also visible in Fig. $3 b$ resulting in ghost targets outside the room.

To eliminate the multi-path effects and denoise the data, we apply the MESI algorithm that exploits sparsity in the time-domain (Fig. 3d) and in the image-domain (Fig. 3e). The figures show that MESI successfully eliminates the multipath reflections even from the top wall that is perpendicular to the antenna array. Fig. 3f demonstrates the effectiveness of the MESI algorithm in denoising and reconstructing the image from the subsampled radar array data in Fig. 2c. The imaged result contains significantly less clutter than Fig. $3 \mathrm{c}$ although the top right targets are not identified due to the severe subsampling.

In conclusion, we showed that the MESI algorithm is a highly effective at eliminating wall-clutter and multi-path reflections from TWI data. The algorithm also successfully identifies targets from compressively sampled and noisy measurements. Moreover, the simulation results demonstrate MESI's robustness to model mismatch since the FDTD setup does not necessarily satisfy assumptions A1 and A2. Finally, we note that the performance is best when the sources of the multi-path reflections are parallel to the antenna array.

\section{ACKNOWLEDGMENT}

The authors would like to thank Petros Boufounos, Phillip Orlik, Li Li, and Zafer Sahinoglu for insightful discussions on antenna arrays and through-the-wall-imaging systems.

\section{REFERENCES}

[1] M. G. Amin and F. Ahmad, "Through-the-wall radar imaging: Theory and applications," Radar Signal Processing Section, E-Reference Signal Processing, Elsevier, 2013.

[2] T. S. Ralston, G. L. Charvat, and J. E. Peabody, "Real-time through-wall imaging using an ultrawideband multiple-input multiple-output (MIMO) phased array radar system," in IEEE International Symposium on Phased Array Systems and Technology (ARRAY), 2010, pp. 551-558.

[3] P. Setlur, M. Amin, and F. Ahmad, "Multipath model and exploitation in through-the-wall and urban radar sensing," IEEE Transactions on Geoscience and Remote Sensing, vol. 49, no. 10, pp. 4021-4034, 2011.

[4] P. Setlur, G. Alli, and L. Nuzzo, "Multipath exploitation in through-wall radar imaging via point spread functions," IEEE Transactions on Image Processing, vol. 22, 2013.

[5] P. C. Chang, "Physics-based inverse processing and multi-path exploitation for through-wall radar imaging," Ph.D. dissertation, Ohio State University, 2011.

[6] M. Leigsnering, F. Ahmad, M. G. Amin, and A. M. Zoubir, "Compressive Sensing Based Specular Multi-path Exploitation for Through-the-wall Radar Imaging," in Proc. of the IEEE ICASSP, March 2013.

[7] J. A. Högbom, "Aperture Synthesis with a Non-Regular Distribution of Interferometer Baselines," Astronomy and Astrophysics Supplement Series, vol. 15, pp. 417-426, June 1974. 